\title{
Continuum Derrida Approach to Drift and Diffusivity in Random Media
}

\author{
D S Dean ${ }^{\dagger}$, I T Drummond and R R Horgan \\ Department of Applied Mathematics and Theoretical Physics \\ University of Cambridge \\ Silver St \\ Cambridge, England CB3 9EW \\ †Dipartimento di Fisica and INFN \\ Univesità di Roma La Sapienza \\ P. A. Moro 2, 00185 Roma, Italy
}

November 23, 2018

\begin{abstract}
By means of rather general arguments, based on an approach due to Derrida that makes use of samples of finite size, we analyse the effective diffusivity and drift tensors in certain types of random medium in which the motion of the particles is controlled by molecular diffusion and a local flow field with known statistical properties.

The power of the Derrida method is that it uses the equilibrium probability distribution, that exists for each finite sample, to compute asymptotic behaviour at large times in the infinite medium. In certain cases, where this equilibrium situation is associated with a vanishing microcurrent, our results demonstrate the equality of the renormalization processes for the effective drift and diffusivity tensors. This establishes, for those cases, a Ward identity previously verified only to two-loop order in perturbation theory in certain models.

The technique can be applied also to media in which the diffusivity exhibits spatial fluctuations. We derive a simple relationship between the effective diffusivity in this case and that for an associated gradient drift problem that provides an interesting constraint on previously conjectured results.
\end{abstract}

DAMTP-96-80 


\section{Introduction}

Problems of flow and diffusion in random environments are of great interest for many reasons both mathematical and physical [1, 2, 3]. Possible applications range from turbulent diffusion [4]-9] to ionic and molecular diffusion in the presence of trapped ions, dislocations and other impurities [10].

A typical continuum model, that represents some aspects of these physical systems, comprises a particle in a $D$ dimensional space subject to molecular diffusion, $\kappa_{i j}$, together with a local drift velocity, $\mathbf{u}(\mathbf{x})$. The motion of a particle at position $\mathbf{x}$ is given by

$$
\dot{\mathbf{x}}=\mathbf{u}(\mathbf{x})+\mathbf{w}(t)
$$

where $\mathbf{w}(t)$ is a white noise process that satisfies

$$
\left\langle w_{i}(t)\right\rangle=0 \quad \text { and } \quad\left\langle w_{i}(t) w_{j}\left(t^{\prime}\right)\right\rangle=2 \kappa_{i j} \delta\left(t-t^{\prime}\right)
$$

The flow field $\mathbf{u}(\mathbf{x})$ is time independent and assumed to have certain statistical properties, the details of which depend on the particular model. However we do assume that the system is homogeneous so that, for example,

$$
\left\langle u_{i}(\mathbf{x}) u_{j}\left(\mathbf{x}^{\prime}\right)\right\rangle=\Delta_{i j}\left(\mathbf{x}-\mathbf{x}^{\prime}\right)
$$

and $\langle\mathbf{u}(\mathbf{x})\rangle$ is independent of position. Frequently the statistics of the velocity field are assumed (for simplicity) to be Gaussian in character so that only the mean velocity and its two-point correlator are required to determine all correlators. We use angle brackets to indicate an average over the white noise ensemble and/or over the ensemble of samples of the medium as appropriate. The intended version of the averaging procedure will be clear from the context.

The computational problem we address then, is to use the above statistical information on the model to deduce the effective drift and diffusivity that control the motion of the particles at large distances and times - the bulk properties of the medium. There has been a great deal of work on this problem [4]-[9], [1]-16]. In this paper we develop a continuum version of an approach by Derrida [1, 17] that he applied to lattice models in order to elucidate relationships that may exist between the effective long range parameters of the theory, one example of which is the "Einstein relation" between drift and diffusivity [2, 3]. 


\section{Derrida Argument for the Continuum}

The idea behind Derrida's argument [17], which is drawn from statistical physics, is that the bulk properties of an infinite medium can be captured in a sample of finite size provided this size is much larger than the typical correlation lengths of the statistical fluctuations of the velocity field $\mathbf{u}(\mathbf{x})$. The size of the sample provides an infra-red cut-off, to use the language of field theory. The assumption being made is that the effective parameters are insensitive to the infra-red cut-off in a limit in which it becomes very large. Of course the presence of important long range correlations would lead to a dependence of the effective parameters on the sample size (and geometry). These are circumstances in which we would expect to see anomalous diffusion [1].

Another important issue in relation to the removal of the infra-red cut-off is the extent to which this limit commutes with the large time limit invoked in the definition of bulk quantities [1] . We do not address this point mathematically in this paper but rely, for the moment, on physical intuition. The commutation of these limits is a very important issue both from a mathematical and a physical point of view and should be examined more thoroughly.

We will assume, therefore, that the medium is represented by a velocity field $u_{i}(\mathbf{x})$ that is defined over a basis region $\Omega$ and that space is tessellated by this region under displacements $\mathbf{l}$ that lie in a Bravais lattice $B$. For the purposes of visualization it is sufficient to suppose that $\Omega$ is cube of side $L$. The velocity field $u_{i}(\mathbf{x})$ then satisfies

$$
u_{i}(\mathbf{x}+\mathbf{l})=u_{i}(\mathbf{x})
$$

and we assume that $\Omega$ which contains the origin, is very large relative to the basic correlation length in $u_{i}(\mathbf{x})$.

A blob of particles is released from the origin at $t=0$. They move independently according to eq(1) and are free to cross the boundaries of $\Omega$ and it's replicates. The resulting probability distribution, $P(\mathbf{x}, t)$, satisfies

$$
\frac{\partial}{\partial t} P(\mathbf{x}, t)=\partial_{i}\left(\kappa_{i j} \partial_{j}-u_{i}(\mathbf{x})\right) P(\mathbf{x}, t) .
$$

All the moments of the distribution can be obtained from the moment generating function of the distribution

$$
Z(\mathbf{k})=\int d^{D} \mathbf{x} e^{-i \mathbf{k} \cdot \mathbf{x}} P(\mathbf{x}, t)
$$


The technical step suggested by Derrida is to re-write this integral over all space as a sum over replicates of the basis region $\Omega$, thus

$$
Z(\mathbf{k})=\int_{\Omega} d^{D} \mathbf{x} \sum_{\mathbf{l}} e^{-i \mathbf{k} \cdot(\mathbf{x}+\mathbf{l})} P(\mathbf{x}+\mathbf{l}, t)
$$

That is

$$
Z(\mathbf{k})=\int_{\Omega} d^{D} \mathbf{x} W(\mathbf{x}, t)=\int_{\Omega} d^{D} \mathbf{x} e^{-i \mathbf{k} \cdot \mathbf{x}} R(\mathbf{x}, t)
$$

where

$$
R(\mathbf{k}, \mathbf{x}, t)=\sum_{\mathbf{l}} e^{-i \mathbf{k} . \mathbf{l}} P(\mathbf{x}+\mathbf{l}, t)
$$

and

$$
W(\mathbf{k}, \mathbf{x}, t)=e^{-i \mathbf{k} \cdot \mathbf{x}} R(\mathbf{k}, \mathbf{x}, t)
$$

The probability distribution can be reconstructed as

$$
P(\mathbf{x}, t)=\int \frac{d^{D} \mathbf{k}}{(2 \pi)^{D}} e^{i \mathbf{k} \cdot \mathbf{x}} Z(\mathbf{k})
$$

Note that there is no restriction on the integration range of $\mathbf{k}$.

Making use of the periodicity properties of the velocity field we can show that

$$
\frac{\partial}{\partial t} R(\mathbf{k}, \mathbf{x}, t)=\partial_{i}\left(\kappa_{i j} \partial_{j}-u_{i}(\mathbf{x})\right) R(\mathbf{k}, \mathbf{x}, t)
$$

where $R(\mathbf{x}, t)$ satisfies the boundary condition

$$
R(\mathbf{k}, \mathbf{x}+\mathbf{a}, t)=e^{i \mathbf{k} \cdot \mathbf{a}} R(\mathbf{k}, \mathbf{x}, t)
$$

with a an element of the Bravais lattice.

As $t \rightarrow \infty$ we expect that time dependence will disappear. In that limit

$$
\partial_{i}\left(\kappa_{i j} \partial_{j}-u_{i}(\mathbf{x})\right) R(\mathbf{k}, \mathbf{x}, t)=0
$$

This implies that for large $t$

$$
R(\mathbf{k}, \mathbf{x}, t) \propto Q_{0}(\mathbf{x})
$$

where $Q_{0}(\mathbf{x})$ is the static probability distribution in $\Omega$. The advantage of the Derrida method is precisely that one may make use of this static equilibrium distribution to evaluate asymptotic behaviour.

However $Q_{0}(\mathbf{x})$ is periodic under displacements in the Bravais lattice and, except for the case $\mathbf{k}=0, R(\mathbf{k}, \mathbf{x}, t)$ is not periodic. Hence we must have

$$
R(0, \mathbf{x}, t) \rightarrow Q_{0}(\mathbf{x})
$$


and

$$
R(\mathbf{k}, \mathbf{x}, t) \rightarrow 0
$$

when $\mathbf{k} \neq 0$. All of this is entirely consistent with continuity in $\mathbf{k}$ at finite time. The equation satisfied by $W(\mathbf{k}, \mathbf{x}, t)$, which is periodic on the Bravais lattice, is

$$
\frac{\partial}{\partial t} W(\mathbf{k}, \mathbf{x}, t)=\left(\partial_{i}+i k_{i}\right)\left(\kappa_{i j}\left(\partial_{j}+i k_{j}\right)-u_{i}(\mathbf{x})\right) W(\mathbf{k}, \mathbf{x}, t)
$$

When $\mathbf{k}=0$ we know that the operator on the right has an eigenfunction with a zero eigenvalue, namely $Q_{0}(\mathbf{x})$, the stationary probability distribution. When $\mathbf{k} \neq 0$ this eigenfunction is perturbed and the eigenvalue is moved away from zero. We denote the eigenfunction by $Q(\mathbf{x})$ and the eigenvalue by $-\mu(\mathbf{k})$ so that $\mu(0)=0$ and

$$
\left(\partial_{i}+i k_{i}\right)\left(\kappa_{i j}\left(\partial_{j}+i k_{j}\right)-u_{i}(\mathbf{x})\right) Q(\mathbf{x})=-\mu(\mathbf{k}) Q(\mathbf{x})
$$

An examination of the (trivial) case with constant velocity field suggests that near $\mathbf{k}=0$ there is a gap in the spectrum of this operator of magnitude $\sim(2 \pi / L)^{2}$ provided $|\mathbf{k}|$ much less than $2 \pi / L$. We assume this to be true and conclude that up to exponentially damped corrections that are $O\left(e^{-(2 \pi / L)^{2} t}\right)$, the solution of eq(17) in which we are interested is

$$
W(\mathbf{k}, \mathbf{x}, t)=Q(\mathbf{x}) e^{-\mu(\mathbf{k}) t}
$$

We now impose the normalization condition

$$
\int_{\Omega} d^{D} \mathbf{x} Q(\mathbf{x})=1
$$

to obtain the resulting formula for the moment generating function is

$$
Z(\mathbf{k})=e^{-\mu(\mathbf{k}) t}
$$

where we have taken into account eq(20) . The corresponding formula for the probability distribution in the unbounded (but tessellated) space is therefore

$$
P(\mathbf{x}, t)=\int \frac{d^{D} \mathbf{k}}{(2 \pi)^{D}} e^{i \mathbf{k} \cdot \mathbf{x}-\mu(\mathbf{k}) t}
$$

For large $t$ the asymptotic form of $P(\mathbf{x}, t)$ is determined by the terms in $\mu(\mathbf{k})$ up to $O\left(\mathbf{k}^{2}\right)$. The remaining terms of higher order determine the approach to this asymptotic form in inverse powers of $\sqrt{t}$ in the general case.

It should be noted that on the basis of the above argument the asymptotic behaviour is guaranteed to set in only when $t$ is so large that only values of $|\mathbf{k}|$ much less than 
$2 \pi / L$ yield important contributions to the integral in eq(22). This means that we are computing asymptotic behaviour that becomes apparent after the probability distribution of the particle has spread over more than one replicate of $\Omega$. It is the physical assumption of the Derrida method that, when the samples are sufficiently large and there is no anomalous diffusive behaviour, this asymptotic behaviour sets in effectively much earlier and therefore there is no change to the estimated bulk diffusivity and drift when the infra-red cutoff is removed. Proving this mathematically remains to be done. Any such proof should also tell us about the physically important issue of how effects on different scales in the problem interact with one another.

\section{Asymptotic Behaviour}

We can calculate these terms in $\mu(\mathbf{k})$ by perturbation theory. Set

$$
Q(\mathbf{x})=Q_{0}(\mathbf{x})+Q_{1}(\mathbf{x})+Q_{2}(\mathbf{x})+\cdots,
$$

and

$$
\mu(\mathbf{k})=\mu_{1}(\mathbf{k})+\mu_{2}(\mathbf{k})+\cdots,
$$

where the suffix on a term indicates its order in powers of $\mathbf{k}$. Note that there is no term $\mu_{0}$ and that the normalization of $Q_{0}(\mathbf{x})$ means that

$$
\int d^{D} \mathbf{x} Q_{1}(\mathbf{x})=\int d^{D} \mathbf{x} Q_{2}(\mathbf{x})=0
$$

This means that $Q_{1}, Q_{2}$ and all higher corrections lie in what we will refer to as the zero weight subspace. We can obtain the equations for the higher corrections by expanding both sides of eq(18) in powers of $\mathbf{k}$. We find

$$
\partial_{l}\left(\kappa_{l m} \partial_{m}-u_{l}(\mathbf{x})\right) Q_{1}(\mathbf{x})+i k_{l}\left(2 \kappa_{l m} \partial_{m}-u_{l}(\mathbf{x})\right) Q_{0}(\mathbf{x})=-\mu_{1}(\mathbf{k}) Q_{0}(\mathbf{x})
$$

and

$$
\begin{aligned}
\partial_{l}\left(\kappa_{l m} \partial_{m}-u_{l}(\mathbf{x})\right) Q_{2}(\mathbf{x})+i k_{l}\left(2 \kappa_{l m} \partial_{m}-u_{l}(\mathbf{x})\right) Q_{1}(\mathbf{x})-k_{l} k_{m} \kappa_{l m} Q_{0}(x) \\
=-\mu_{2}(\mathbf{k}) Q_{0}(\mathbf{x})-\mu_{1}(\mathbf{k}) Q_{1}(\mathbf{x}) .
\end{aligned}
$$

For completeness we note that the general recursion formula $(n \neq 2)$ is

$$
\partial_{l}\left(\kappa_{l m} \partial_{m}-u_{l}(\mathbf{x})\right) Q_{n}(\mathbf{x})+i k_{l}\left(2 \kappa_{l m} \partial_{m}-u_{l}(\mathbf{x})\right) Q_{n-1}(\mathbf{x})
$$




$$
=-\mu_{n}(\mathbf{k}) Q_{0}(\mathbf{x})-\cdots-\left(\mu_{2}(\mathbf{k})-k_{l} k_{m} \kappa_{l m}\right) Q_{n-2}(\mathbf{x})-\mu_{1}(\mathbf{k}) Q_{n-1}(\mathbf{x}) .
$$

After integration over the base cell $\Omega$, eq(26) yields

$$
\mu_{1}(\mathbf{k})=i k_{l} \int_{\Omega} d^{D} \mathbf{x} u_{l}(\mathbf{x}) Q_{0}(\mathbf{x})=i k_{l} \bar{u}_{l}
$$

where $\bar{u}_{l}$ is the average value of the velocity in the stationary probability distribution $Q_{0}(\mathbf{x})$. Note that the vanishing, under integration, of the gradient terms is assured by the periodicity and continuity of the random field.

To compute the next correction we need the Green's function $G\left(\mathbf{x}, \mathbf{x}^{\prime}\right)$ that is the inverse of the operator

$$
H=\partial_{l}\left(\kappa_{l m} \partial_{m}-u_{l}(\mathbf{x})\right)
$$

in the zero weight subspace. The existence of this Green's function depends on our physically plausible assumption that the kernel of $H$ is one-dimensional and is therefore spanned by $Q_{0}(\mathbf{x})$.

We have then

$$
\partial_{l}\left(\kappa_{l m} \partial_{m}-u_{l}(\mathbf{x})\right) G\left(\mathbf{x}, \mathbf{x}^{\prime}\right)=-\delta\left(\mathbf{x}, \mathbf{x}^{\prime}\right) \equiv-\delta\left(\mathbf{x}-\mathbf{x}^{\prime}\right)+\frac{1}{V},
$$

where $V$ is the volume of $\Omega$. It follows then that

$$
Q_{1}(\mathbf{x})=i k_{l} \int_{\Omega} d^{D} \mathbf{x}^{\prime} G\left(\mathbf{x}, \mathbf{x}^{\prime}\right)\left(2 \kappa_{l m} \partial_{m}^{\prime}-\left(u_{l}\left(\mathbf{x}^{\prime}\right)-\overline{u_{l}}\right)\right) Q_{0}\left(\mathbf{x}^{\prime}\right)
$$

The evaluation of $\mu_{2}$ is achieved by integrating eq(27) with respect to $\mathbf{x}$ over $\Omega$. We have

$$
\mu_{2}=k_{l} \kappa_{l m} k_{m}+i k_{l} \int_{\Omega} d^{D} \mathbf{x} u_{l}(\mathbf{x}) Q_{1}(\mathbf{x})
$$

That is

$$
\mu_{2}=k_{l} \kappa_{l m} k_{m}-k_{l} k_{m} \int_{\Omega} d^{D} \mathbf{x} d^{D} \mathbf{x}^{\prime} u_{l}(\mathbf{x}) G\left(\mathbf{x}, \mathbf{x}^{\prime}\right)\left(2 \kappa_{m n} \partial_{n}^{\prime}-\left(u_{m}\left(\mathbf{x}^{\prime}\right)-\bar{u}_{m}\right)\right) Q_{0}\left(\mathbf{x}^{\prime}\right)
$$

These results tell us that the mean velocity of the cloud is $\bar{u}_{i}$ and the effective diffusivity tensor is

$$
\kappa_{l m}^{\mathrm{e}}=\kappa_{l m}-\frac{1}{2}\left\{\int_{\Omega} d^{D} \mathbf{x} d^{D} \mathbf{x}^{\prime} \tilde{u}_{l}(\mathbf{x}) G\left(\mathbf{x}, \mathbf{x}^{\prime}\right)\left(2 \kappa_{m n} \partial_{n}^{\prime}-\tilde{u}_{m}\left(\mathbf{x}^{\prime}\right)\right) Q_{0}\left(\mathbf{x}^{\prime}\right)+(l \leftrightarrow m)\right\}
$$

where we have set

$$
\tilde{u}_{l}(\mathbf{x})=u_{l}(\mathbf{x})-\bar{u}_{l}(\mathbf{x})
$$

and used the result

$$
\int_{\Omega} d^{D} \mathbf{x} G\left(\mathbf{x}, \mathbf{x}^{\prime}\right)=0 .
$$




\section{Gradient Flow}

A case of particular interest is that of gradient flow where the local drift depends linearly on the gradient of a scalar field. Thus

$$
u_{l}(\mathbf{x})=\lambda_{l m} \partial_{m} \phi(\mathbf{x})
$$

In this case the equilibrium microcurrent is

$$
J_{l}(\mathbf{x})=-\left(\kappa_{l m} \partial_{m}-\lambda_{l m} \partial_{m} \phi(\mathbf{x})\right) Q_{0}(\mathbf{x})
$$

Within this class of models an important case arises for which the drift and diffusivity tensors are proportional to one another,

$$
\lambda_{l m}=\tau \kappa_{l m}
$$

In this case the microcurrent vanishes in equilibrium and the static probability distribution satisfies

$$
\partial_{l} \kappa_{l m}\left(\partial_{l}-\tau \partial_{l} \phi(\mathbf{x})\right) Q_{0}(\mathbf{x})=0
$$

That is

$$
Q_{0}(\mathbf{x})=N e^{\tau \phi(\mathbf{x})}
$$

where $\mathrm{N}$ is a normalization constant. It is also the case that

$$
\bar{u}_{l}(\mathbf{x})=\int_{\Omega} d^{D} \mathbf{x} \tau \kappa_{l m} \partial_{m} \phi(\mathbf{x}) Q_{0}(\mathbf{x})=0 .
$$

It follows that $\tilde{\mathbf{u}}(\mathbf{x})=\mathbf{u}(\mathbf{x})$.

The formula for the effective diffusivity, eq(34), becomes

$$
\kappa_{l m}^{\mathrm{e}}=\kappa_{l m}-\int_{\Omega} d^{D} \mathbf{x} d^{D} \mathbf{x}^{\prime} u_{l}(\mathbf{x}) G\left(\mathbf{x}, \mathbf{x}^{\prime}\right) u_{m}\left(\mathbf{x}^{\prime}\right) Q_{0}\left(\mathbf{x}^{\prime}\right)
$$

which is symmetrical in $l$ and $m$. Since $G\left(\mathbf{x}, \mathbf{x}^{\prime}\right) Q_{0}\left(\mathbf{x}^{\prime}\right)$ is a positive operator we see that the tendency is for the random behaviour of the medium to reduce the effective diffusivity in a given direction relative to the microscopic value. This is consistent with the idea that the random fluctuations of the field $\phi(\mathbf{x})$ represent a trapping mechanism tending to hold the particle near the bottoms of potential wells. 


\section{External Gradient}

To investigate the computation of the effective drift we add a constant external gradient to the fluctuating gradient. We have

$$
u_{l}(\mathbf{x})=\tau \kappa_{l m}\left(\partial_{m} \phi(\mathbf{x})+g_{m}\right)
$$

The static probability distribution now satisfies

$$
\partial_{l} \kappa_{l m}\left(\partial_{m}-\tau\left(\partial_{m} \phi(\mathbf{x})+g_{m}\right)\right) Q_{0}^{g}(\mathbf{x})=0
$$

When $g_{m}$ is small we can compute $\bar{u}_{l}$ to lowest order in $g_{m}$. If we set

$$
Q_{0}^{g}(\mathbf{x})=Q_{0}(\mathbf{x})+q_{1}(\mathbf{x})+\cdots,
$$

then we easily find

$$
q_{1}(\mathbf{x})=-\int d^{D} \mathbf{x}^{\prime} G\left(\mathbf{x}, \mathbf{x}^{\prime}\right) \tau \kappa_{l m} \partial_{l}^{\prime} Q_{0}\left(\mathbf{x}^{\prime}\right) g_{m}
$$

The mean velocity is, to $O(g)$,

$$
\bar{u}_{l}=\tau\left(\kappa_{l m}-\int d^{D} \mathbf{x} d^{D} \mathbf{x}^{\prime} \tau \kappa_{l n} \partial_{n} \phi(\mathbf{x}) G\left(\mathbf{x}, \mathbf{x}^{\prime}\right) \kappa_{r m} \partial_{r}^{\prime} Q_{0}\left(\mathbf{x}^{\prime}\right)\right) g_{m}
$$

From eq(29) we find

$$
\bar{u}_{l}=\tau\left(\kappa_{l m}-\int d^{D} \mathbf{x} d^{D} \mathbf{x}^{\prime} u_{l}(\mathbf{x}) G\left(\mathbf{x}, \mathbf{x}^{\prime}\right) u_{m}\left(\mathbf{x}^{\prime}\right) Q_{0}\left(\mathbf{x}^{\prime}\right)\right) g_{m} .
$$

This is also the result we obtained in a previous paper. It shows that the drift tensor is renormalized in the same way as the diffusivity tensor if we start from a situation where they are proportional at the microscopic level. Because this proportionality of the effective drift and diffusivity tensors holds sample by sample it also holds after averaging over the ensemble of random media. The precise nature of the statistics of the velocity field are not important for this result. There is no requirement for Gaussian statistics, for example.

The result will also hold in the limit in which sample size becomes infinite, assuming the absence of very long range correlations. Consequently the result guarantees the Ward identity that was discussed in previous work [14, 15, 16]. It remains remarkable that the direct proof of this identity, even in perturbation theory, is so unobvious that it has only been achieved at the two-loop level. It would be very interesting to obtain a 
proof of the Ward identity directly in the infinite medium to all orders in perturbation theory.

The results confirm those of Kravtsov, Lerner and Yudson [2, 3] in the case of isotropic diffusion, drift and (Gaussian) statistics. Our result shows that the only important point, as inferred by these authors, is the existence of the potential controlled stationary distribution with a vanishing microcurrent. In fact by an appropriate linear transformation on the independent variables $\mathbf{x}$, the diffusivity tensor can be rendered isotropic and simultaneously, the drift tensor rendered diagonal. Aside from the question of the statistics of the field $\phi(\mathbf{x})$ therefore, the more general problem considered here is mathematically identical to the isotropic case.

\section{Incompressible Flow}

Much work has been done on incompressible flow [4, 5, 6]. The velocity field satisfies

$$
\partial_{l} u_{l}(\mathbf{x})=0
$$

The diffusion equation for the probability distribution can can be written

$$
\frac{\partial}{\partial t} P(\mathbf{x}, t)=\left(\kappa_{i j} \partial_{j}-u_{i}(\mathbf{x})\right) \partial_{i} P(\mathbf{x}, t) .
$$

It is then easy to see that the static distribution satisfies

$$
\partial_{i} Q_{0}(\mathbf{x})=0
$$

This implies that $Q_{0}=1 / V$. The role played by the mean velocity in this case is rather trivial. It is just the centre of mass motion of the incompressible fluid. For this reason, as noted in [2, 3], it is never renormalized by the fluctuations in the velocity field.

In an appropriate reference frame then, the mean velocity is zero. That is

$$
\int_{\Omega} d^{D} \mathbf{x} u_{l}(\mathbf{x})=0
$$

From eq(34) it follows that

$$
\kappa_{l m}^{\mathrm{e}}=\kappa_{l m}+\int_{\Omega} d^{D} \mathbf{x} d^{D} \mathbf{x}^{\prime} u_{l}(\mathbf{x}) G\left(\mathbf{x}, \mathbf{x}^{\prime}\right) u_{m}\left(\mathbf{x}^{\prime}\right) / V .
$$

This is similar to eq(43) but with a change of sign for the correction to the bare diffusivity. This implies that the effect of the incompressible flow is to enhance the 
diffusivity. In fact in practical cases the contribution of turbulent dispersion to the effective diffusivity dominates that of molecular diffusion by many orders of magnitude. In this case it is reasonable to consider a limit in which the molecular diffusivity goes to zero. This is not possible in the gradient flow case where the particle will simply become trapped at the first stationary point it encounters in the potential.

\section{$7 \quad$ Large Sample Limit}

For completeness we briefly discuss the large sample limit which we have assumed is innocuous in the sense that appropriate spatial averages are equivalent to ensemble averages. A simple example is the normalization of the static probability distribution in the case of gradient flow. We have

$$
Q_{0}(\mathbf{x})=N e^{\tau \phi(\mathbf{x})}
$$

Consider

$$
X=\int_{\Omega} d^{D} \mathbf{x} e^{\tau \phi(\mathbf{x})}
$$

The ensemble average, in the case of Gaussian statistics, is

$$
\langle X\rangle=\int_{\Omega} d^{D} \mathbf{x}\left\langle e^{\tau \phi(\mathbf{x})}\right\rangle=\int_{\Omega} d^{D} \mathbf{x} e^{\frac{1}{2} \tau^{2} \Delta(0)}=V e^{\frac{1}{2} \tau^{2} \Delta(0)}
$$

where $\Delta\left(\mathbf{x}-\mathbf{x}^{\prime}\right)=\left\langle\phi(\mathbf{x}) \phi\left(\mathbf{x}^{\prime}\right)\right\rangle$. We also have

$$
\left\langle X^{2}\right\rangle=\int_{\Omega} d^{D} \mathbf{x} d^{D} \mathbf{x}^{\prime}\left\langle e^{\tau \phi(\mathbf{x})+\tau \phi\left(\mathbf{x}^{\prime}\right)}\right\rangle=\int_{\Omega} d^{D} \mathbf{x} d^{D} \mathbf{x}^{\prime} e^{\tau^{2} \Delta(0)+\tau^{2} \Delta\left(\mathbf{x}-\mathbf{x}^{\prime}\right)} .
$$

The variance of $X$ is therefore

$$
\sigma^{2}(X)=\int_{\Omega} d^{D} \mathbf{x} d^{D} \mathbf{x}^{\prime} e^{\tau^{2} \Delta(0)}\left[e^{\tau^{2} \Delta\left(\mathbf{x}-\mathbf{x}^{\prime}\right)}-1\right]=V e^{\tau^{2} \Delta(0)} \int_{\Omega} d^{D} \mathbf{x}\left[e^{\tau^{2} \Delta(\mathbf{x})}-1\right] .
$$

It follows that in the limit of large samples in which $V \rightarrow \infty$, both the mean and variance of $X$ are $O(V)$. That is for large $V$ we may use the result

$$
X=V e^{\frac{1}{2} \tau^{2} \Delta(0)}(1+\xi)
$$

where $\xi$ has zero mean and $\sigma^{2}(\xi)=O(1 / V)$, which suggests that $\xi$ can be ignored in the large sample limit. This tells us that in this limit we can use the normalization

$$
N=e^{-\frac{1}{2} \tau^{2} \Delta(0)} / V
$$


A careful analysis of perturbation theory suggests that similar results hold for other appropriate quantities. In particular we expect for large samples,

$$
\mu(\mathbf{k})=\langle\mu(\mathbf{k})\rangle+O(1 / \sqrt{V})
$$

Which implies that the infinite medium effective probability distribution is

$$
\mathcal{P}(\mathbf{x}, t)=\int \frac{d^{D} \mathbf{k}}{(2 \pi)^{D}}\left\langle e^{i \mathbf{k} \cdot \mathbf{x}-\mu(\mathbf{k}) t}\right\rangle=\int \frac{d^{D} \mathbf{k}}{(2 \pi)^{D}} e^{i \mathbf{k} \cdot \mathbf{x}-\langle\mu(\mathbf{k})\rangle t} .
$$

This result was assumed in our discussion above.

\section{Spatially Varying Diffusivity}

So far we have applied the Derrida argument to models for which the diffusivity tensor is constant in space. Clearly the argument can be generalised to accommodate spatial fluctuations in the diffusivity. We therefore make the replacement

$$
\kappa_{i j} \rightarrow \kappa_{i j}(\mathbf{x})
$$

where

$$
\kappa_{i j}(\mathbf{x}+\mathbf{l})=\kappa_{i j}(\mathbf{x})
$$

and $\mathbf{l}$ is an element of the Bravais lattice. Of course we assume that the correlation length of the fluctuations in the diffusivity tensor is very much smaller than the size of the cell $\Omega$. For simplicity we will assume also that the local drift term vanishes since it will not be needed for the application we wish to make. The physical realizations of this problem are in the computation of the bulk versions of the diffusivity tensor, permeability tensor or permittivity tensor in the corresponding diffusion, fluid flow or electrodynamical problems [1] together with the bulk response to the appropriate external fields.

If we adopt the diffusion model, the probability density function for a particle in the medium satisfies the equation

$$
\frac{\partial}{\partial t} P(\mathbf{x}, t)=\partial_{i} \kappa_{i j}(\mathbf{x}) \partial_{j} P(\mathbf{x}, t)
$$

It is immediately obvious that the static solution is

$$
Q_{0}(\mathbf{x})=1 / V
$$


In this case the application of the Derrida technique, along the lines discussed above, leads us to consider the perturbation analysis of the equation

$$
H(\mathbf{k}) Q(\mathbf{x})=-\mu(\mathbf{k}) Q(\mathbf{x})
$$

where

$$
H(\mathbf{k})=\left(\partial_{i}+i k_{i}\right) \kappa_{i j}(\mathbf{x})\left(\partial_{j}+i k_{j}\right)
$$

and $\mu(\mathbf{k})$ is the eigenvalue that vanishes when $\mathbf{k}=0$. In the standard way we write $\mu(\mathbf{k})=\mu_{1}(\mathbf{k})+\mu_{2}(\mathbf{k})+\cdots$ and $Q(\mathbf{x})=Q_{0}(\mathbf{x})+Q_{1}(\mathbf{x})+\cdots$. However in the present case it is easily established that $\mu_{1}(\mathbf{k})=0$ and that

$$
Q_{1}(\mathbf{x})=i k_{i} \int_{\Omega} d^{D} \mathbf{x}^{\prime} G\left(\mathbf{x}, \mathbf{x}^{\prime}\right) \partial_{j}^{\prime} \kappa_{i j}\left(\mathbf{x}^{\prime}\right) Q_{0}
$$

where the Green's function $G\left(\mathbf{x}, \mathbf{x}^{\prime}\right)$ satisfies

$$
\partial_{i} \kappa_{i j}(\mathbf{x}) \partial_{j} G\left(\mathbf{x}, \mathbf{x}^{\prime}\right)=-\delta\left(\mathbf{x}, \mathbf{x}^{\prime}\right)
$$

A quick way to compute $\mu_{2}(\mathbf{k})$ is to integrate eq 68 over $\Omega$ to obtain

$$
\mu(\mathbf{k})=-i k_{i} \int_{\Omega} d^{D} \mathbf{x} \kappa_{i j}(\mathbf{x})\left(\partial_{j}+i k_{j}\right) Q(\mathbf{x})
$$

On picking out the $O\left(k^{2}\right)$ term from this equation, we find

$$
\mu_{2}(\mathbf{k})=\bar{\kappa}_{i j} k_{i} k_{j}-i k_{i} \int_{\Omega} d^{D} \mathbf{x} \kappa_{i j}(\mathbf{x}) \partial_{j} Q_{1}(\mathbf{x})
$$

where $\bar{\kappa}_{i j}$ is the spatial average of the diffusivity tensor,

$$
\bar{\kappa}_{i j}=\frac{1}{V} \int_{\Omega} d^{D} \mathbf{x} \kappa_{i j}(\mathbf{x})
$$

From eq $(\sqrt[70]{ })$ we find

$$
\mu_{2}(\mathbf{k})=\bar{\kappa}_{i j} k_{i} k_{j}+k_{i} k_{j} \int_{\Omega} d^{D} \mathbf{x} d^{D} \mathbf{x}^{\prime} \kappa_{i l}(\mathbf{x}) \partial_{l} G\left(\mathbf{x}, \mathbf{x}^{\prime}\right) \partial_{m}^{\prime} \kappa_{j m}\left(\mathbf{x}^{\prime}\right)
$$

and after integration by parts

$$
\mu_{2}(\mathbf{k})=\bar{\kappa}_{i j} k_{i} k_{j}-\frac{k_{i} k_{j}}{V} \int_{\Omega} d^{D} \mathbf{x} d^{D} \mathbf{x}^{\prime}\left[\partial_{l} \kappa_{i l}(\mathbf{x})\right] G\left(\mathbf{x}, \mathbf{x}^{\prime}\right) \partial_{m}^{\prime} \kappa_{j m}\left(\mathbf{x}^{\prime}\right)
$$

Hence we have obtained an expression for $\kappa_{i j}^{\mathrm{e}}$.

We now restrict ourselves to the isotropic case where $\kappa_{i j}(\mathbf{x})=\kappa_{0} e^{\tau \phi(\mathbf{x})} \delta_{i j}$. In the literature the case of lognormal statistics where $\phi(\mathbf{x})$ is a zero mean Gaussian field 
with two point function $\Delta\left(\left|\mathbf{x}-\mathbf{x}^{\prime}\right|\right)$ has been widely studied. It can also be related, by means of the appropriate Green's functions, to a diffusion problem with constant diffusivity $\kappa_{0}$ and drift parameter $\lambda_{0}=\tau \kappa_{0}$. The Green's function for this latter problem, $G^{\text {grad }}\left(\mathbf{x}, \mathbf{x}^{\prime}\right)$, satisfies

$$
\partial_{i} \kappa_{0}\left(\partial_{i}-\tau \partial_{i} \phi(\mathbf{x})\right) G^{g r a d}\left(\mathbf{x}, \mathbf{x}^{\prime}\right)=-\delta\left(\mathbf{x}, \mathbf{x}^{\prime}\right)
$$

whereas $G\left(\mathbf{x}, \mathbf{x}^{\prime}\right)$ satisfies

$$
\partial_{i} \kappa_{0} e^{\tau \phi(\mathbf{x})} \partial_{i} G\left(\mathbf{x}, \mathbf{x}^{\prime}\right)=-\delta\left(\mathbf{x}, \mathbf{x}^{\prime}\right)
$$

It is easy to see now that the Green's function for the original diffusivity problem is related to that for the gradient problem by

$$
G\left(\mathbf{x}, \mathbf{x}^{\prime}\right)=e^{-\tau \phi(\mathbf{x})} G^{g r a d}\left(\mathbf{x}, \mathbf{x}^{\prime}\right)
$$

Substituting this into (76) we get

$$
\mu_{2}(\mathbf{k})=\bar{\kappa} k^{2}-\frac{k_{i} k_{j}}{V} \int_{\Omega} d^{D} \mathbf{x} d^{D} \mathbf{x}^{\prime}\left[\partial_{i} \kappa_{0} e^{\tau \phi(\mathbf{x})}\right] e^{-\tau \phi(\mathbf{x})} G^{g r a d}\left(\mathbf{x}, \mathbf{x}^{\prime}\right) \partial_{j}^{\prime} \kappa_{0} e^{\tau \phi\left(\mathbf{x}^{\prime}\right)}
$$

where

$$
\bar{\kappa}=\frac{1}{V} \int d^{D} \mathbf{x} \kappa_{0} e^{\tau \phi(\mathbf{x})}
$$

If now we take the ensemble average $\bar{\kappa}$ becomes

$$
\bar{\kappa}=\frac{1}{V} \int d^{D} \mathbf{x} \kappa_{0}\left\langle e^{\tau \phi(\mathbf{x})}\right\rangle=\kappa_{0} e^{\frac{1}{2} \tau^{2} \Delta(0)}
$$

and obtain the result

$$
\kappa^{\mathrm{e}}=\kappa_{0} e^{\frac{1}{2} \tau^{2} \Delta(0)}-\frac{1}{D} \tau \kappa_{0}^{2} \frac{k_{i} k_{j}}{V} \int_{\Omega} d^{D} \mathbf{x} d^{D} \mathbf{x}^{\prime}\left\langle\left[\partial_{i} \phi(\mathbf{x})\right] G^{g r a d}\left(\mathbf{x}, \mathbf{x}^{\prime}\right) \partial_{i}^{\prime} e^{\tau \phi\left(\mathbf{x}^{\prime}\right)}\right\rangle,
$$

We can compare this with the result of substituting $\kappa_{i j}=\kappa_{0} \delta_{i j}$ and $u_{i}(\mathbf{x})=\tau \kappa_{0} \partial_{i} \phi(\mathbf{x})$ into eq(43).

$$
\kappa_{l m}^{\mathrm{e}}=\kappa_{0} \delta_{l m}-\tau^{2} \kappa_{0}^{2} \int_{\Omega} d^{D} \mathbf{x} d^{D} \mathbf{x}^{\prime}\left[\partial_{l} \phi(\mathbf{x})\right] G^{g r a d}\left(\mathbf{x}, \mathbf{x}^{\prime}\right)\left[\partial_{m} \phi\left(\mathbf{x}^{\prime}\right)\right] Q_{0}\left(\mathbf{x}^{\prime}\right)
$$

where $Q_{0}(\mathbf{x})=N e^{\tau \phi(\mathbf{x})}$. On taking the ensemble average and using the isotropy we obtain for the effective diffusivity in the gradient flow case

$$
\kappa_{\text {grad }}^{\mathrm{e}}=\kappa_{0}-\frac{1}{D} \tau^{2} \kappa_{0}^{2} \frac{1}{V e^{\frac{1}{2} \tau^{2} \Delta(0)}} \int_{\Omega} d^{D} \mathbf{x} d^{D} \mathbf{x}^{\prime}\left[\partial_{m} \phi(\mathbf{x})\right] G^{\text {grad }}\left(\mathbf{x}, \mathbf{x}^{\prime}\right)\left[\partial_{m} \phi\left(\mathbf{x}^{\prime}\right)\right] e^{\tau \phi\left(\mathbf{x}^{\prime}\right)}
$$


The result is that

$$
\kappa^{\mathrm{e}} / \bar{\kappa}=\kappa_{\text {grad }}^{\mathrm{e}} / \kappa_{0} .
$$

This means that the ratio of the bulk diffusivity to the (ensemble or spatial) average of the local diffusivity in the fluctuating diffusivity problem is equal to the ratio of the bulk diffusivity to the local diffusivity in the associated gradient drift problem. For the case of a lognormal diffusivity then we have

$$
\kappa^{\mathrm{e}}=e^{\frac{1}{2} \tau^{2} \Delta(0)} \kappa_{\text {grad }}^{\mathrm{e}}
$$

The consequences of this simple result are quite significant. In one dimension both problems are easily solved exactly and it is known that

$$
\kappa^{\mathrm{e}}=\kappa_{0} e^{-\frac{1}{2} \tau^{2} \Delta(0)}
$$

and

$$
\kappa_{\text {grad }}^{\mathrm{e}}=\kappa_{0} e^{-\tau^{2} \Delta(0)} .
$$

Hence our result is of course consistent with the well known exact results in one dimension.

In two dimensions however the random diffusivity case may be solved exactly via duality arguments 19, 20, 21], giving simply $\kappa^{\mathrm{e}}=1$. This means that $\kappa_{\text {grad }}^{\mathrm{e}}=e^{-\frac{1}{2} \tau^{2} \Delta(0)}$ giving a new exact result for the isotropic gradient flow problem in two dimensions.

In fact,these results in one and two dimensions are consistent with a renormalisation group calculation in D dimensions for the isotropic gradient flow case [13, 12] that gives

$$
\kappa_{\text {grad }}^{\mathrm{e}}=\kappa_{0} e^{-\tau^{2} \Delta(0) / D}
$$

Although the renormalization group calculation is based on certain plausible though unproved assumptions, it maintains the equality of the drift and diffusion renormalization factors and has been accurately verified in three dimension by numerical simulation over a considerable range in the value of this factor. It has been verified by analytical calculation to two loop-order in perturbation theory. The numerical simulation suggests however that it remains accurate beyond this order.

$\mathrm{Eq}(\sqrt{86})$ allows us to relate these renormalization group results to the diffusivity problem. There has been for some time, a speculation in the literature, for example see [18], again verified to two-loop order in perturbation theory, that in the diffusivity problem

$$
\kappa^{\mathrm{e}}=\kappa_{0} e^{\left(\frac{1}{2}-\frac{1}{D}\right) \tau^{2} \Delta(0)} .
$$


Because eq(86) is an exact relation it shows that they stand or fall together. Recently [22, 23] it has been shown that, in three dimensions, at third loop order eq(91) breaks down and corrections explicitly depending on the spatial structure of the correlation function appear. This would mean therefore that the formula (90) similarly fails at three loop order in three dimensions. So far it has not proved possible to analyse this problem to three loops directly. However a detailed analysis relating the two calculations should make further progress possible in the gradient drift problem. It is worth noting that even if a breakdown of the simple formulae is established, the success of eq(90) in describing the results of numerical simulations suggests that it is not necessarily a severe one.

\section{Conclusions}

We have developed the Derrida technique [17], which was originally introduced for lattice models [1, 17], to the continuum case. It shows very conveniently the relationship between the effective drift and diffusivity tensors in general random media. In particular, for gradient flow models, it makes clear that when the microscopic drift and diffusivity tensors are proportional to one another the corresponding effective tensors show precisely the same proportionality [13]. This guarantees the validity, under very general statistical regimes, of a Ward identity relating the effective propagator and vertex in wave vector space, derived up to the two-loop level in previous papers [14, 15, 16]. It ties in with the ideas behind the Einstein relation of the drift [2, [3] and diffusivity by a temperature factor. Here the role of temperature is played by the inverse of the proportionality factor $\tau$. Of course this may have no direct connection with the real temperature of the sample.

Applied to incompressible flow the method recovers immediately the non-renormalization of the mean flow by fluctuations in the velocity field. This is an obvious result on physical grounds and confirms previous work on the renormalization group approach to anomalous diffusion [2, [3].

The method can also be applied to the problem of a random medium with a spatially fluctuating diffusivity. It reveals for the isotropic log-normal case the relationship of the bulk diffusivity with that for a related gradient flow model. This relation is confirmed in the case of one and two dimensional models and provides a constraint on the predictions of models in higher dimensions. The violation of simple results for the bulk diffusivity in one model being tied to possible failure of simple results in the 
other.

In principle the formulae derived from the Derrida method also allows the calculation of higher order terms that control the approach to asymptotic behaviour of the various moments of the probability distribution. However the exploitation of such results would require a proper elucidation of the commutation of the large sample and large time limits. This is an important mathematical problem that remains to be investigated.

\section{References}

[1] J.-P. Bouchaud and A. Georges, Phys. Rep. 195, 128 - 293, 1990

[2] V.E. Kravtsov, I.V. Lerner and V.I. Yudson, Zh. Eksp. Teor. Fiz.,91, 569 - 586, 1986 Sov. Phys. JETP, 64, 1986

[3] V.E. Kravtsov, I.V. Lerner and V.I. Yudson, J. Phys. A: Math. Gen., 119, 203 206, 1986

[4] R.H. Kraichnan, J. Fluid Mech., 77, 753, 1976

[5] R. Phythian and W.D. Curtis, J. Fluid Mech., 89, 241 - 250, 1978

[6] I.T. Drummond, S. Duane and R.R. Horgan, J. Fluid Mech., 138, 75, 1984

[7] P.R. King J. Phys. A: Math Gen., 20, 3935, 1987

[8] I.T. Drummond and R.R. Horgan, J. Phys. A: Math Gen., 20, 4661, 1987

[9] I.T. Drummond and R.R. Horgan, J. Phys. A: Math. Gen., 22, 3205, 1990

[10] J.A. Sousa Goncalves, R.L. Portsmouth, P. Alexander and L.F. Gladden, J. Phys. Chem., 99, 3317 - 3325, 1995

[11] D.S. Dean Stochastic Dynamics, PhD Thesis, University of Cambridge, 1993

[12] M.W. Deem and D. Chandler, Journal of Stat. Phys., 76, 911, 1994

[13] D.S. Dean, I.T. Drummond and R.R. Horgan, J. Phys. A: Math Gen., 27, 5135, 1994

[14] D.S. Dean, I.T. Drummond and R.R. Horgan, J. Phys. A: Math Gen., 28, 1235, 1995 
[15] D.S. Dean, I.T. Drummond and R.R. Horgan, J. Phys. A: Math. Gen., 28, 60136025, 1995

[16] D.S. Dean, I.T. Drummond and R R Horgan, Renormalization of Drift and Diffusivity in Random Gradient Flows, Submitted to J. Phys. A: Math. Gen.

[17] B. Derrida, J. Stat. Phys., 31, 433, 1983

[18] G. Matheron, Eléments pour une theorie des milieux poreux (Masson, Paris), 1967

[19] J. B. Keller, J. Math. Phys., bf 5, 548, 1964

[20] A. M. Dykhne, Sov. Phys. JETP, 32, 63, 1971

[21] A. M. Dykhne, Sov. Phys. JETP, 32, 348, 1971

[22] A. De Wit Physics of Fluids, 7, 11, 2553, 1995

[23] B. Abramovich and P. Indelman, J. Phys. A: Math. Gen. 28, 693, 1995 